\title{
Étude comparative de la qualité de la symbiose (Anabaena azollae, Azolla caroliniana), du compost et du NPK sur la croissance végétative et le rendement de la tomate (Lycopersicon esculentum mill. Solanacée) à Daloa (Côte d'Ivoire)
}

\author{
GROGA Noel1, DIOMANDE Massé², BEUGRE Grah Avit Maxwell², OUATTARA Yaya², AKAFFOU Doffou \\ Selastique ${ }^{1}$ \\ 'Laboratoire d'amélioration de la production agricole, UFR Agrofresterie, Université Jean Lorougnon Guède Daloa Côte \\ d'Ivoire ; \\ 2Laboratoire d'agro valorisation, UFR Agrofresterie, Université jean Lorougnon Guède Daloa Côte d'Ivoire ; Auteur \\ correspondant : GROGA Noel1BP 150 Daloa, email : groga7@yahoo.fr
}

Original submitted in on $3^{\text {rd }}$ May 2018. Published online at www.m.elewa.org on 30 http://dx.doi.org/10.4314/jab.v129i1.4

\section{RESUME}

Objectif : La présente étude a pour objectif de comparer la qualité fertilisante de l'algue verte (Azolla caroliniana), du compost et du NPK appliqués à la tomate dans la zone de Daloa (Côte d'Ivoire). Les paramètres évalués sont la croissance végétative et le rendement de ces tomates.

Méthodologie et résultats : L'essai est réalisé en bloc complet randomisé de quatre traitements (Azolla caroliniana, compost, NPK et le Témoin) en quatre répétitions. Les données sont collectées sur le cycle végétatif de cultures. Les traitements ont été comparés suivants les paramètres végétatifs : hauteur de tiges (ht), diamètre du collet (dc), longueur de feuilles (If) et le rendement (r). Hormis les traitements à base de NPK avaient reçus $200 \mathrm{~kg} / \mathrm{ha}$ pour NPK et $100 \mathrm{~kg} / \mathrm{h}$ a pour l'urée, les traitements sont fertilisés à la même dose de fumier (30 t/ha). Les résultats indiquent l'Azolla caroliniana statiquement diffèrent. La moyenne de (ht) est de $49,37 \pm 0,55 \mathrm{~cm}$ avec l'Azolla caroliniana contre $37 \pm 1 \mathrm{~cm}$ pour le NPK, $34,57 \pm 2,2$ pour le compost et 22,95 $\pm 0,24$ pour le témoin, sauf la croissance du (dc) où la plus petite différence n'a pas été significative. Le ( $d c)$ est de $0,71 \pm 0,08$; contre $0,57 \pm 0,03$ pour le NPK, $0,55 \pm 0,08$ pour le compost et $0,42 \pm 0,07$ pour le témoin. Le rendement a été important avec l'Azolla caroliniana (26,13 $\pm 0,86$ tha de tomate) pour une densité de 37500 plants/ha.

Conclusion et application des résultats : Cette étude montre que l'Azolla caroliniana donne un rendement important moyennant une densité forte, restaure une bonne fertilité du sol et permet d'accroître la productivité de la culture de tomate.

Mots clés : la tomate, Azolla caroliniana, NPK, compost, paramètres végétatifs, rendement. 


\section{ABSTRACT}

Objective : The present study aims to compare the fertilizing quality of green algae (Azolla caroliniana), compost and NPK applied to tomato in the Daloa area (Côte d'Ivoire). The parameters evaluated are the vegetative growth and yield of these tomatoes.

Methodology and results: The trial is performed as a randomized, complete block of four treatments (Azolla caroliniana, compost, NPK and Control) in four replicates. Data are collected on the vegetative cycle of crops. The treatments were compared according to the vegetative parameters : stem height ( $\mathrm{ht}$ ), neck diameter (dc), leaf length (If) and yield (r). Except NPK treatments received $200 \mathrm{~kg} / \mathrm{ha}$ for NPK and $100 \mathrm{~kg} / \mathrm{ha}$ for urea, treatments are fertilized at the same manure rate (30 t/ ha). The results indicate Azolla caroliniana statically differ. The average of (ht) is $49.37 \pm 0.55 \mathrm{~cm}$ with Azolla caroliniana against $37 \pm 1 \mathrm{~cm}$ for NPK, $34.57 \pm 2.2$ for compost and $22.95 \pm 0.24$ for control, except the growth of (dc) where the smallest difference was not significant. (Dc) is $0.71 \pm 0.08$; against $0.57 \pm 0.03$ for NPK, $0.55 \pm 0.08$ for compost and $0.42 \pm 0.07$ for the control. Yield was high with Azolla caroliniana ( $26.13 \pm 0.86 \mathrm{t} / \mathrm{ha}$ tomato) at a density of 37500 plants / ha. Conclusion and application of results: This study shows that Azolla caroliniana gives a high yield with a high density, restores good soil fertility and increases the productivity of the tomato crop.

Keywords: tomato, Azolla caroliniana, NPK, compost, vegetative parameters, yield

\section{INTRODUCTION}

Les cultures maraîchères sont devenues ces dernières années une activité répondant de façon efficace à la demande alimentaire urbaine (Singbo et al, 2004). La culture de tomate (Lycopersicum esculentum) constitue une activité lucrative pour de nombreux producteurs (Hanson, 2001), à cause de sa richesse en minéraux et représente une source prédominante en antioxydants bénéfiques pour la santé comme les caroténoïdes (lycopène et $\beta$ carotène) non synthétisés par les êtres humains et les vitamines, (acide ascorbique et a-tocophérol) impliqués dans le processus de détoxification des cellules et aident à la prévention de nombreux cancers (Abidi et al., 2017). Aussi, ces composés bioactifs diététiques contenus dans les tomates réduiraient le risque de problèmes de santé chroniques tels que les maladies cardio-vasculaires, l'hypertension et les deux types de diabète. Selon les statistiques de l'organisation des Nations unies pour l'alimentation et l'agriculture (FAO,1980), la production mondiale de tomates s'élevait en 2013 à 164.5 millions de tonnes pour une surface de 4,77 millions d'hectares, soit un rendement moyen de 34.5 tha. En Côte d'Ivoire, la production annuelle fluctue entre 22000 et 35000 tonnes (Sangaré et al., 2009). En effet, les besoins en tomates estimés à plus de 100000 tonnes ne sont couverts qu'aux deux tiers par la production locale. Ce faible rendement, favorisé par un mauvais développement des plantes, est dû à une petite application des engrais minéraux, à la faible fertilité du sol, à la forte pression parasitaire et à la sensibilité aux climats chauds et humides (Témé et al., 1995 ; Dugué, 1993 ; Anderson, 1992). La faible utilisation des engrais n'augure pas à atteindre la sécurité alimentaire ou à inverser le grave appauvrissement des sols en nutriments en Afrique. Une utilisation substantielle d'engrais est un pré requis dans le succès des efforts d'amélioration de la productivité agricole et partant, de l'accroissement de la production et des revenus ${ }^{22}$ agricoles en Afrique (Anonyme 1, 2013). Aucun pays du monde n'a atteint une croissance agricole substantielle sans utiliser d'engrais. Les maraîchers reviennent sur le même sol toute l'année, cela impose la restauration permanente de sa fertilité. En effet, les besoins nutritionnels de la tomate sont énormes surtout en $\mathrm{N}$ et $\mathrm{K}$. Les engrais minéraux coûtent chers et ne sont pas à la portée de tous les paysans. Le revenu modique des maraîchers dû à la faible production, suite aux travaux accomplis encore manuellement, ne leur permet pas de s'en procurer en quantité suffisante. Le coût élevé d'engrais minéraux couplé à leur faible accessibilité constitue un facteur limitant en culture maraichère, particulièrement en culture de tomate (Kitabala et al, 2016). En plus l'utilisation des engrais minéraux conduit à des pertes d'azote, tel que les émissions ammoniacales; à des risques importants 
de lessivage vers les nappes phréatiques et les cours d'eau ; à des intoxications. Afin de limiter les risques économiques, environnementaux, et sociaux liés à l'utilisation des engrais minéraux tel que le NPK, l'agriculteur doit viser les engrais organiques qui pourront s'avérer moins couteux et bénéfiques. Ils sont une source importante d'éléments nutritifs et peuvent être utilisés pour élever la productivité des sols pauvres en nutriments majeurs. La piste de recherche est orientée vers les biofertilisants tel que le compost et les plantes fertilisantes: les

\section{MATERIEL ET METHODES}

Présentation de la zone d'étude : Le site de l'étude est à Daloa, localité de Côte d'Ivoire dans la région du Haut Sassandra entre le $7^{\circ} 00^{\prime} \mathrm{N}$ et $6^{\circ} 35^{\prime} \mathrm{W}$. Cette région a une superficie de $15200 \mathrm{~km}^{2}$ pour une population légumineuses, les non-légumineuses dont l'Azolla. L'objectif de ce travail est de comparer la qualité fertilisante de l'Azolla caroliniana (fougère aquatique vivant en symbiose avec une bactérie Anabaena azollae), du compost et du NPK sur la croissance végétative puis le rendement de la tomate (Lycopersicum esculentum) dans la zone de Daloa (Côte d'Ivoire). Les résultats de cette étude permettront de faire des propositions de nouveaux bios fertilisants efficaces aux paysans.

estimée à 1.430 .960 habitants (Anonyme 2, 2014). La parcelle expérimentale est située au sein de l'Université Jean Lorougnon Guédé (figure 1).

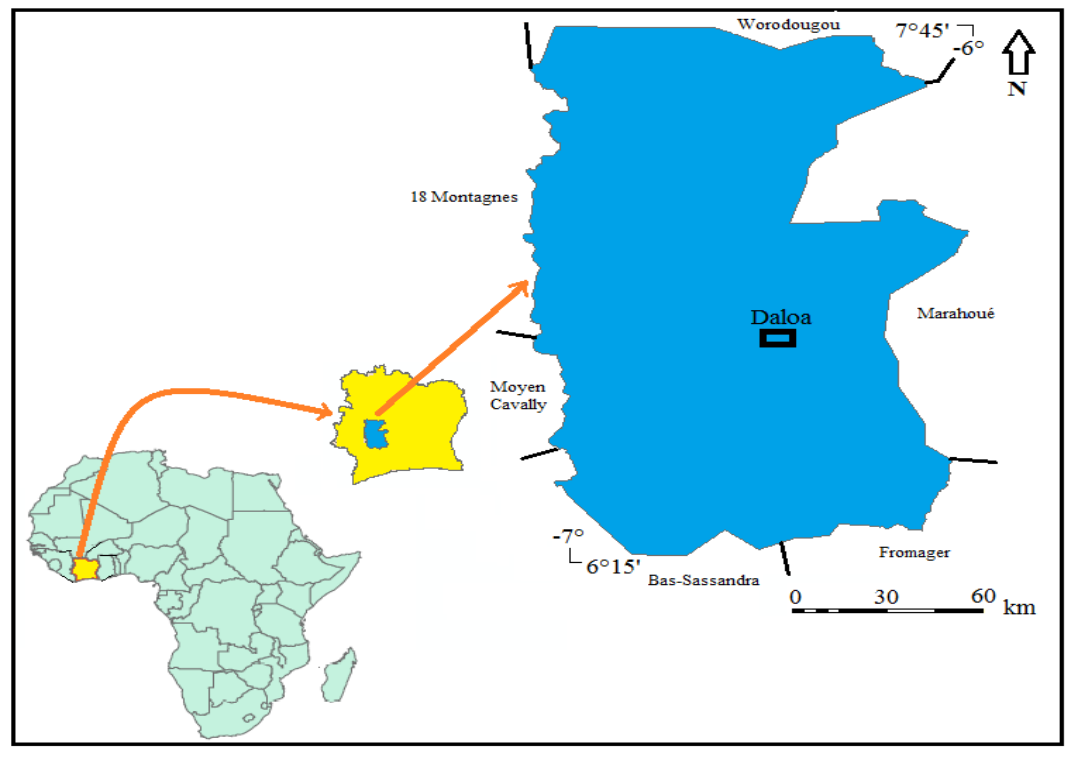

Figure 1: Zone d'étude (Daloa, Côte d'Ivoire)

Matériel végétal : Le matériel végétal est composé d'une semence de tomate hybride de variété topaze $F 1$ venu de SEMIVOIRE. II s'agit d'une variété améliorée de la société TECHNISEM. Elle a été choisie pour, son taux de germination qui est de $85 \%$, une pureté de $99 \%$, le poids élevé de ces fruits et sa rigueur qui assure des récoltes étalées et de haute qualité. Les biofertilisants utilisés pendant l'expérimentation sont l'Azolla caroliniana (fougère aquatique) et le compost. L'engrais minéral NPK 122222 et l'urée commerciale ont aussi été utilisés. Plusieurs techniques et matériaux de compostages existent. Cependant nos travaux ont nécessité, de la fiente de poulet, de la sciure de bois blanc, d'eau de robinet, de la pulpe de café et du charbon de bois. II faut au moins 90 jours pour que le compost soit bien décomposé et utilisable. Toutefois, il faut un mélange du tas par semaine.

\section{Méthodes}

Mise en place de la culture d'Azolla caroliniana : Elle s'est effectuée dans une fosse, elle a consisté à ajouter 125 litres d'eau dans une fosse de $3 \mathrm{~m}$ de longueur, $1 \mathrm{~m}$ de largeur et de 30 à $45 \mathrm{~m}$ de profondeur, Des sachets plastiques bleu ont été disposés à l'intérieur des fosses afin d'éviter la fuite d'eau. Les fosses ainsi préparées sont 
exposées à la lumière du jour. La fiente de poulet contient des éléments nutritifs comme fertilisant. Pour ce fait, nous avons ajouté $0.5 \mathrm{~kg}$ de fiente de poulet (Galus galus) dans 5 I d'eau de robinet. L'ensemble (fiente et eau) a séjourné pendant 2 jours à l'ombre. Enfin le mélange a été filtré pour recueillir le liquide. Ensuite on ajoute 6 litres de fiente liquide dans cette fosse et enfin on inocule 125 $\mathrm{g}$ d'Azolla. Nous avons recueilli le poids de la production après 3 semaines.

Mise en place de la Culture de la tomate : La mise en place s'est faite de façon manuelle, la parcelle a été défrichée à la machette ensuite labouré à une profondeur de 30 à $35 \mathrm{~cm}$. Après, a suivi la mise en place de 12 planches, chacune avait une moyenne de $6 \mathrm{~m}$ de longueur $3 \mathrm{~m}$ de largeur et de $30 \mathrm{~cm}$ de hauteur. La distance entre ces planches était de $1 \mathrm{~m}$ en moyenne. Des bacs de $1 \mathrm{~m}^{2}$ de surface et de hauteur $20 \mathrm{~cm}$ ont servi à la mise en place de la pépinière. Elle a consisté à mettre une quantité de sol prélevé au sein de l'Université Jean Lorougnon Guédé dans les bacs. Le semis a été réalisé en ligne continu de façon homogène. La quantité de semence de tomate utilisée était de $30 \mathrm{~g}$. Les semences ont été couvertes avec de la terre et ensuite de la paille pendant trois jours. Après le semis, la pépinière a été arrosée régulièrement. Vingt-un jours après semis en pépinière, les plants ont été repiqués dans les planches. Le repiquage se fait sur 3 lignes, et a un écartement de $0.4 \mathrm{~m}$ entre deux plants et $0.6 \mathrm{~m}$ entre les lignes.

Fertilisation des planches : La fertilisation a été faite avec des engrais organiques (Azolla caroliniana, compost) et minérale (NPK + urée). L'épandage a été effectué sur toutes les parcelles à raison de 30 tha pour les engrais organiques et $200 \mathrm{~kg} / \mathrm{ha}$ pour NPK et 100 $\mathrm{kg} / \mathrm{ha}$ pour l'urée. La fiente de poulet, utilisée comme engrais de fond (toutes les parcelles) a été incorporée au sol quatre semaines avant le repiquage, à raison de 20 t/ha.

Entretien et Protection de la parcelle : Pendant toute la période culturale, le champ expérimental était arrosé deux fois par jours, matin et soir avec une quantité d'environ 36 litre d'eau de robinet. L'entretien a porté sur le sarclage et le désherbage. Le désherbage a été effectué 2 fois, le premier désherbage au $15^{\mathrm{e}}$ jour après repiquage et le deuxième un mois après repiquage. Le sarclage a été effectué deux mois après le repiquage. Les cultures maraîchères sont des cultures très sensibles. Les traitements phytosanitaires les protègent contre les maladies (champignon, virus), les insectes et araignées et les nématodes (ANADER, 2015). Pour cela nous avons utilisé le Decis, à raison de 1 litre par hectare contre les insectes et le Manèbe $10 \mathrm{~g} / \mathrm{m}^{2}$ contre les champignons.

Dispositif expérimental : Le dispositif 'expérimental est un bloc complet randomisé à trois sous blocs. Chacun d'eux est subdivisé en 4 parcelles élémentaires de $2 \mathrm{mx}$ $6 m$ soit $12 m^{2}$. Les sous blocs sont distants les uns des autres de $1 \mathrm{~m}$. L'écartement entre les parcelles élémentaires a été de $1 \mathrm{~m}$. L'essai a été conduit sur une superficie de $1125 \mathrm{~m}^{2}$ (Figure 2). 


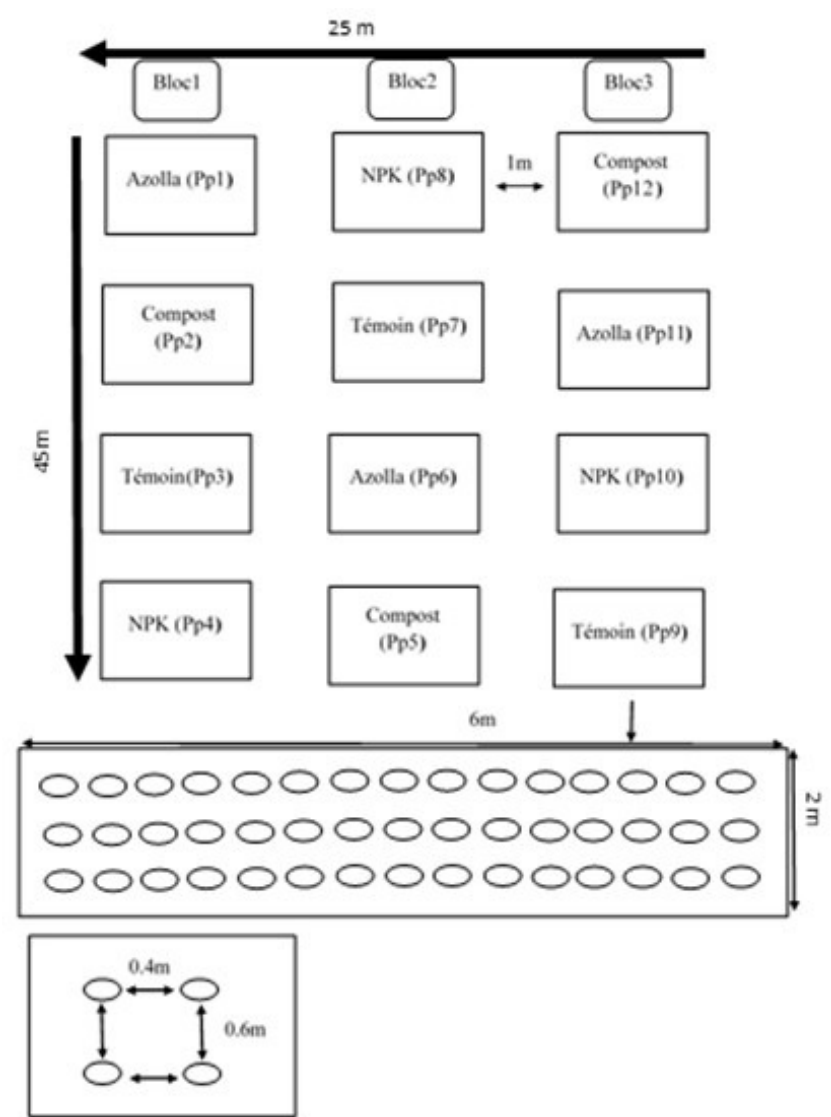

Figure 2 : Dispositif expérimental ; (Pp : Planche à production)

Observation et mesures des paramètres: Des observations et mesure du diamètre au collet, la taille de la tige, la longueur des feuilles ont été effectuées à 3 reprises, on a $T_{0}$ temps des premières observations et mesure, soit le $35^{\mathrm{e}}$ jour du cycle végétatif. $T_{1}$ temps des deuxième observations et mesure, soit le $49 \mathrm{e}$ jour $\mathrm{du}$ cycle végétatif. $T_{2}$ temps des troisième observations et mesure, soit le $65^{\mathrm{e}}$ jour du cycle végétatif. Les composantes du rendement ont été déterminées à partir de la masse des fruits par plants au cours de la récolte. $A$ la fin des récoltes, la masse moyenne par traitement permet de déterminer le rendement avec une densité de 37500 plants/ha.

Analyse des données : Les données collectées ont été d'abord saisies dans un tableau à l'aide d'un logiciel Microsoft Excel 2013 et analysées à l'aide du logiciel STATISTICA 7.1. L'ANOVA est réalisée sur les

\section{RESULTATS ET DISCUSSION}

\section{Résultats}

Paramètres physico-chimiques du compost et de la fiente : Les paramètres physicochimiques du compost et de la fiente de poulet ont contribué à l'enrichissement du moyennes des paramètres mesurés (taille de la tige, diamètre du collet, le poids de fruit) et dénombrement (nombre de fruits par plant). Mais avant l'analyse, les données sont soumises à un test de normalité (Shapiro et al., 1968) et d'homogénéité de variance (Brown \& Forsythe, 1974). L'hypothèse testée est par l'analyse de variance celle de la différence nulle entre les traitements,

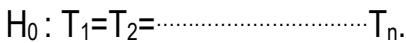

Lorsqu'une différence significative est observée entre les traitements pour un caractère, l'ANOVA est complétée par le test de Tukey. Ce test a permis d'identifier le traitement dont l'effet diffère significativement du ou des autres paramètres et de comparer les moyennes des traitements au seuil de signification $5 \%$. Le niveau de significativité choisi pour les analyses est de $5 \%(P=$ $0,05)$. Le degré de liberté est $n-k$ ou $n$ et $k$ représentent les nombres d'observation et de groupe.

milieu en matières particulaires et dissoutes. Pour évaluer leur efficacité des analyses ont été effectué. Les résultats des analyses ont révélés, un compost de pH 8.1 avec une température de $27,6^{\circ} \mathrm{C}$, la teneur en matière organique 
est de $46 \%$ (\%de matière sèche). Au niveau de la fiente, le $\mathrm{pH}$ du milieu est de 6,47 avec une température de $27,41^{\circ} \mathrm{C}$, la conductivité et la salinité sont respectivement de $15,37 \mu \mathrm{s} / \mathrm{cm}$ et $8,92 \%$, le taux de solide dissous est très important, il est de 9821,14 mg/l (Tableau 1).

Production d'Azolla caroliniana en fonction du temps : Pour une quantité de $125 \mathrm{~g}$ d'Azolla ensemencée au début de l'expérience, une variation de poids a été relevée après 3 semaines de production. Le poids enregistré à une valeur de $7660 \mathrm{~g}$.

Paramètres de croissances et du rendement : Hauteur des tiges de la tomate : Les tailles moyenne des plants de tomates traités avec l'azolla sont plus élevées et significativement différentes par rapport à ceux traitée avec le NPK, le compost, ainsi que les plants témoins. Mais les plants traités avec le NPK n'ont pas eu une différence significative à $T_{0}(10,43 \mathrm{~cm})$ et $T_{1}(22 \mathrm{~cm})$ avec ceux traités à l'azolla à $T_{0}(11,73 \mathrm{~cm})$ et $T_{1}(25,5 \mathrm{~cm})$. Par contre à $T_{2}$, les plants traités avec l'azolla sont significativement différents par rapport à ceux traités avec le NPK. Les moyennes de tailles s'élèvent à $T_{2}$ de 49.37 $\mathrm{cm}$ pour l'azolla contre $37 \mathrm{~cm}$ pour le NPK (Tableau 2). La croissance en hauteur des plants de la tomate fertilisée avec les biofertilisants sont diffèrent de ceux traités avec les fertilisants minéraux (figure 3).

Tableau1 : Caractéristique du compost et de la fiente de poulet

\begin{tabular}{|c|c|c|}
\hline Éléments utilisés & Paramètres & Données (moyennes) \\
\hline \multirow{4}{*}{ Fiente } & $\mathrm{pH}$ & $6,47 \pm 0,01$ \\
\cline { 2 - 3 } & Température ${ }^{\circ} \mathrm{C}$ & $27,41 \pm 0,05$ \\
\cline { 2 - 3 } & Conductivité Électrique (CE) en $\mu \mathrm{s} / \mathrm{cm}$ & $1537 \pm 72,14$ \\
\cline { 2 - 3 } & & $48,92 \pm 2,18$ \\
\hline \multirow{4}{*}{ Compost } & Salinité en $\%$ & $8,1 \pm 0,1$ \\
\cline { 2 - 3 } & $\mathrm{pH}-\mathrm{H}_{2} \mathrm{O}$ & $27,6 \pm 0,5$ \\
\cline { 2 - 3 } & Température $^{\circ} \mathrm{C}$ & $25,60 \pm 2,10$ \\
\cline { 2 - 3 } & Carbone $(\mathrm{C})$ & $2,29 \pm 0,13$ \\
\cline { 2 - 3 } & Azote total (N) & $46 \pm 0,5$ \\
\cline { 2 - 3 }
\end{tabular}

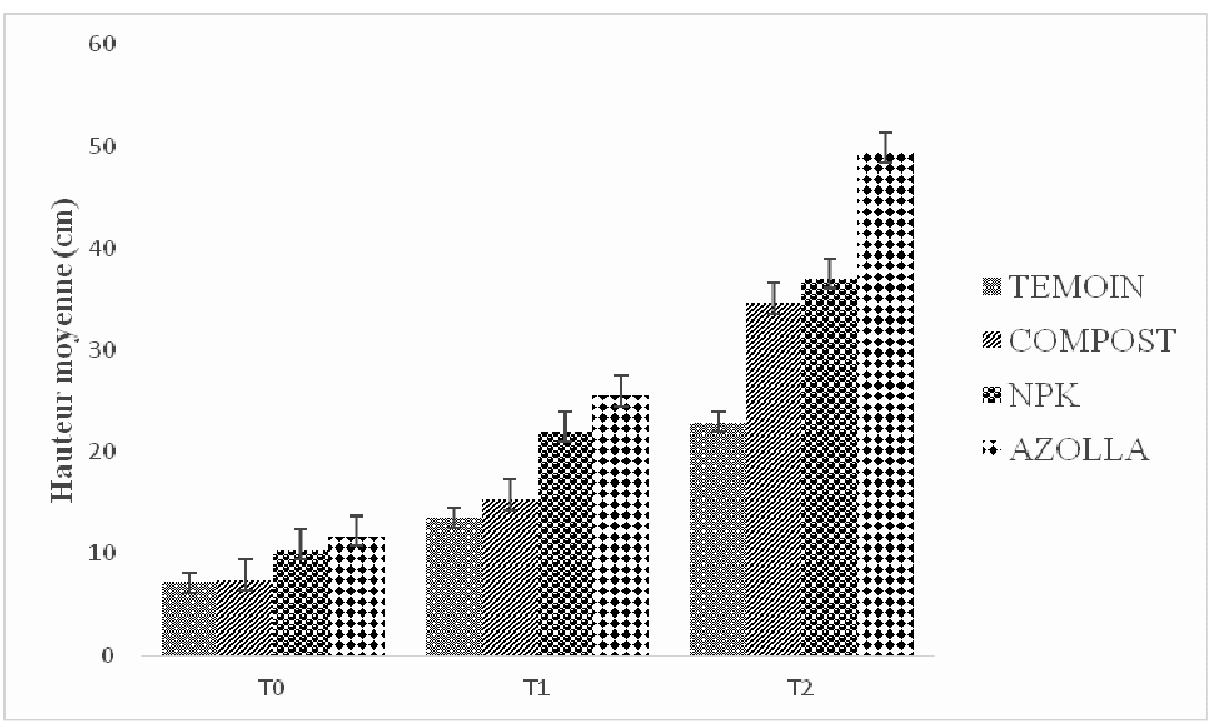

Figure 3 : Diagramme de l'évolution de la hauteur des tiges de la tomate

Diamètre au collet : La croissance du diamètre au collet des plants qui était de $0,23,0,37,0,26,0,4 \mathrm{~cm}$ à $\mathrm{T}_{0}$ respectivement pour le témoin, l'azolla, le compost et le NPK est passée à $0.42,0.71,0.55,0.57 \mathrm{~cm}$ à $T_{2}$. Même si l'analyse statistique révèle qu'il n'existe pas de différence significative à $T_{2}$ entre l'azolla, le compost, et le NPK, l'évolution de l'azolla a été plus effective sur le diamètre au collet par rapport à ceux du compost, le NPK ainsi que 
le témoin (figure 4). D'une manière générale, les différents traitements sur la tomate variété topaze ont un effet sur l'évolution du diamètre au collet.

Longueur des feuilles: Pour les traitements avec l'azolla et le NPK, la longueur moyenne des feuilles sont non seulement les plus élevées mais, elles sont significativement différentes par rapport à ceux traités avec le compost, ainsi que les plants témoins. Pour ces deux traitements on a respectivement $43 \mathrm{~cm}$ et $35,2 \mathrm{~cm}$ à $T_{2}$. Alors qu'on a les plus faibles moyennes pour les plants traités avec le compost de $31,23 \mathrm{~cm}$ à $T_{2}$ ainsi que le témoin avec $26,03 \mathrm{~cm}$ à $T_{2}$. Cette différence est bien observée sur la figure 5 montrant l'évolution de la longueur des feuilles.

Rendement des tomates: Pour les traitements avec l'azolla et le NPK, les rendements sont non seulement les plus élevés, mais ils sont significativement différents par rapport à ceux traités avec le compost, ainsi que les plants témoins. Les plants fertilisés avec l'azolla ont des rendements plus élevés $26,13 t /$ ha par rapport aux plants issus des traitements du NPK 24.7t/ha, du compost 21,52 t/ha ainsi que les plants témoins 5 t/ha (figure 6 ). Selon le traitement appliqué, les rendements moyens diffèrent d'un traitement à l'autre.

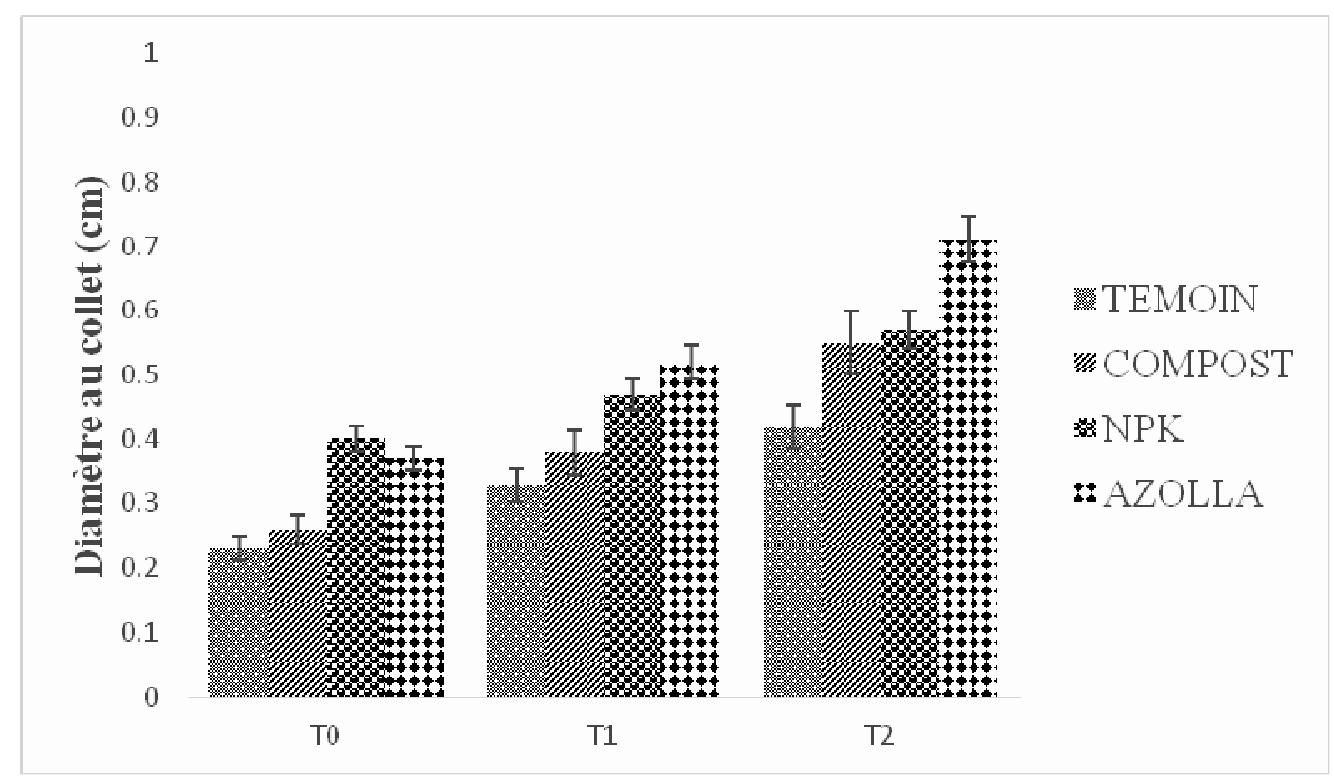

Figure 4 : Diagramme de l'évolution du diamètre au collet

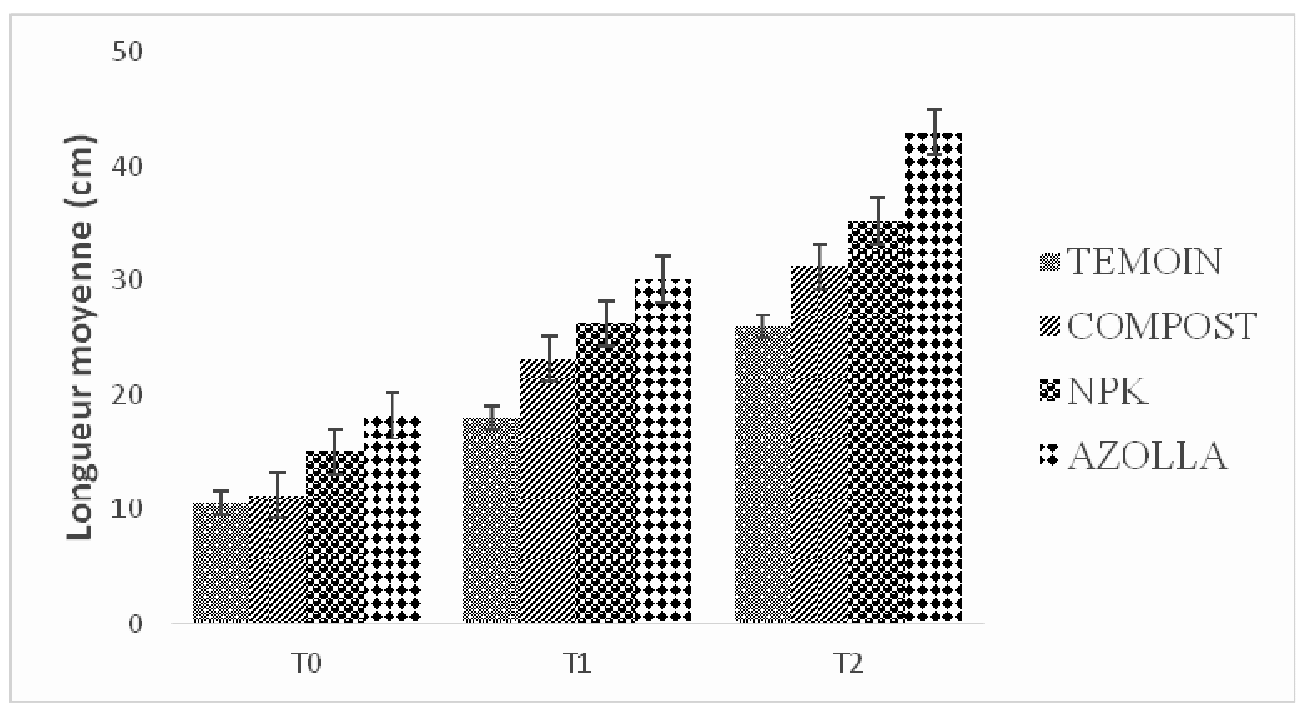

Figure 5 : Diagramme de l'évolution de la longueur des feuilles 


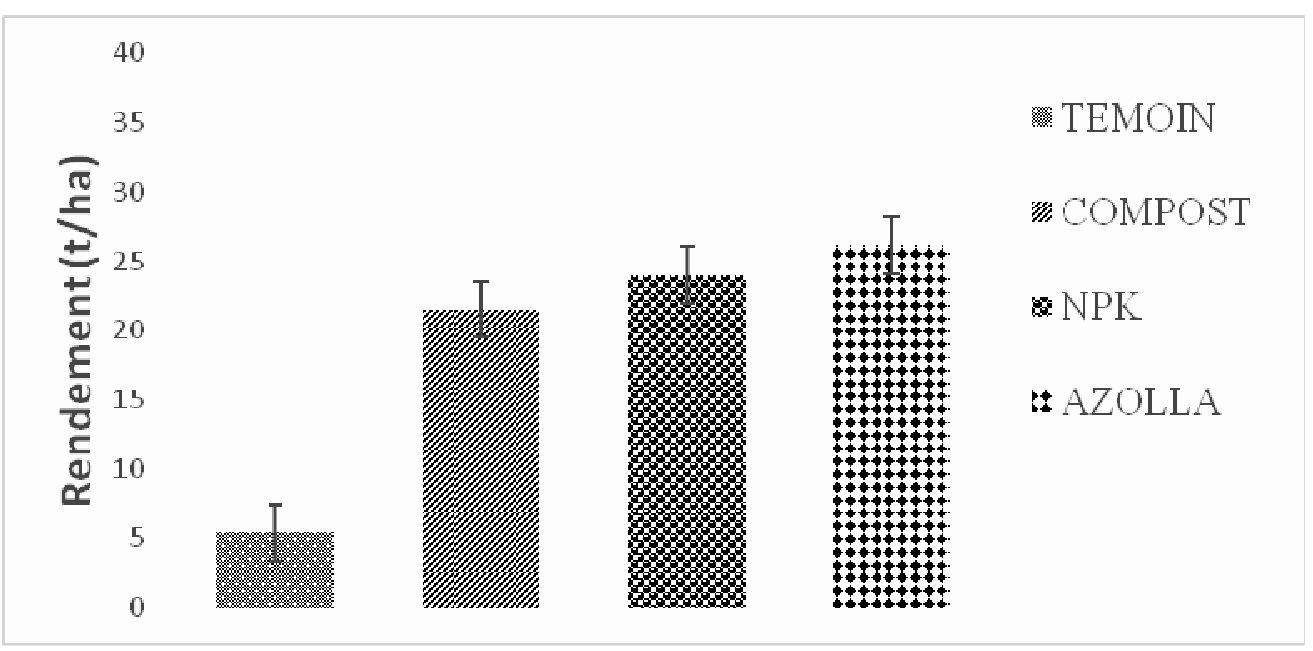

Figure 6 : Diagramme de l'évolution du rendement

\section{DISCUSSION}

Parmi les propriétés d'un substrat idéal, il faut avoir la capacité de tamponner les variations de la salinité et du $\mathrm{pH}$, afin de limiter la Conductivité Électrique (CE) dans une fourchette de 500 à $2000 \mu \mathrm{s} / \mathrm{cm}$ (Morel et al., 2000). La fiente utilisée comme milieu de culture de l'azolla caroliniana présente une salinité de $48,92 \%$. Cette valeur est inférieure à ceux de M'sadak et al., (2013) qui avaient obtenus $94 \%$ de salinité dans un substrat à base de mélange avicole. La teneur élevée en sels affecte la croissance des végétaux qui peut résulter des brûlures aux racines et aux feuillages. Une valeur élevée représente une grande quantité d'ions en solution, ce qui rend plus difficile l'absorption d'eau et d'éléments nutritifs par la plante et peut même brûler les racines. La valeur $\mathrm{du} \mathrm{pH}$ est de 6,17. Cette valeur est conforme à celle dégagé par Kerkeni (2008) qui confirme que les substrats de culture devraient avoir un $\mathrm{pH}$ légèrement acide $(5,2$ à $6,3)$. Si la valeur du pH d'un substrat dépasse 6,5 , des carences peuvent se produire (Comtois \& Légaré, 2004). Un $\mathrm{pH}$ excessif peut entraîner une forte accumulation de $\mathrm{Ca}$ ou de $\mathrm{Na}$, alors qu'un pH inférieur à 5 peut entrainer des phénomènes de toxicité à cause d'une importante accumulation de fer (Ammari et al., 2006). La CE est de $1537 \mu \mathrm{s} / \mathrm{cm}$, conforme à cette fourchette 500 à 2000 $\mu \mathrm{s} / \mathrm{cm}$ cité par (Morel et al., 2000). Soumaré et al., 2002 affirment que les substrats de culture devraient avoir une faible conductivité électrique inférieure à $3000 \mu \mathrm{s} / \mathrm{cm}$. Audelà de cette norme, des répercussions négatives pourraient avoir lieu sur la croissance. Plusieurs paramètres permettre d'évaluer la qualité d'un compost. La teneur en matière organique est un des premiers critères sur lesquels on se base pour juger de la compostabilité d'un produit donné Larbi (2006). Le compost utilisé dans cette étude présente une teneur en matière organique de $46 \%$ ( $\%$ matière sèche $\mathrm{MS}$ ). Le compost utilisé est considéré comme produit mûr car selon Larbi (2006) le compost mûr doit avoir une teneur en matière organique inférieur à $50 \%$ ( $\%$ matière sèche MS). Cette valeur est supérieure à celle trouvée par M'sadak et al., (2013) à base de mélange avicole qui a une teneur en matière organique de $38 \%$ ( $\%$ matière sèche $\mathrm{MS}$ ). L'importance du $\mathrm{pH}$ réside dans le fait qu'il affecte la solubilité des éléments nutritifs dans le sol et dans la solution du sol. Le pH pourrait être un indicateur de la maturité complète d'un substrat. La valeur du pH se situe normalement entre 7 et 8 (Larbi, 2006). À pH acide, le substrat est considéré comme immature (Bernal et al., 1998). Le compost produit, est considérée comme un substrat mature car il présente un $\mathrm{pH}$ de 7,47. Cette valeur est légèrement supérieure au compost à base de tourbe et méthacompost avicole produit par M'sadak et al., (2015) qui a un pH de 7,43. Toutefois, Kerkeni (2008) considère que le $\mathrm{pH}$ n'est pas une mesure très précise de la maturité d'un substrat. La maturité est aussi souvent évaluée par le rapport $\mathrm{C} / \mathrm{N}$. II a été établi qu'un rapport $\mathrm{C} / \mathrm{N}$ voisin de $10-15$ correspond à un compost mature qui peut amender considérablement les sols sur lesquels les plantes tirent leurs substances nutritives. En ce qui concerne le compost que nous avons produit, le rapport $\mathrm{C} / \mathrm{N}$ est de 12,26 ce qui est tout à fait acceptable. Ce qui indique que le compost était mûr. Cette valeur est supérieure au compost à base d'ordure de chou-pommé produit par Gnonzan, (2003) qui a un rapport $\mathrm{C} / \mathrm{N}$ de 11,50 . Pour une quantité de $125 \mathrm{~g}$ d'azolla caroliniana 
ensemencée dans un milieu de culture eau + la fiente de poulet (liquide) au début de l'expérimentation, le poids relevé après 3 semaines a une valeur de $7660 \mathrm{~g}$. Ces résultats sont similaires à ceux de Kouadio (2015), qui avait obtenu pour $100 \mathrm{~g}$ d'azolla filiculoides ensemencé $2979 \mathrm{~g}$ l'azolla filiculoides après 14 jours en milieu de culture renfermant de l'eau + la fiente de poulet. La forte production d'azolla obtenu pourrait s'expliquer par la présence de fiente de poulet, elle met à la disposition de la fougère beaucoup plus d'éléments minéraux nutritifs qui sont favorable à la croissance et au développement de l'azolla. Selon Becking (1979) les carences en éléments ( $\mathrm{P}, \mathrm{K}, \mathrm{Ca}, \mathrm{Mg}$ et $\mathrm{Mn}$ ) et en microéléments ( $\mathrm{Fe}$, Mo, Co) entraînent les diminutions des croissances de l'Azolla. Les paramètres végétatifs étudiés, pour les plants de tomates traités avec azolla sont plus élevés que le compost, le NPK ainsi que le témoin. Le rôle positif de l'azolla sur les paramètres végétatifs, démontré par les résultats obtenus dans cet essai, est confirmé par Kouadio (2015) qui a enregistré des augmentations au niveau de la hauteur du riz, le nombre de talles et le nombre de feuilles du riz comparativement au NPK et le témoin après, l'application d'Azolla filiculoides. Cette croissance se justifie par le faite que l'azolla a une quantité importante d'éléments d'azote et de phosphore, ces éléments sont indispensables à la croissance et au développement des plantes et agissent immédiatement sur le développement du feuillage et sur la production des plantes en culture (Brasset et al, 2005). En effet l'azolla abrite une cyanobactérie du genre Anabaena qui a la propriété de fixer l'azote, c'est-à-dire de transformer l'azote moléculaire de l'atmosphère en azote assimilable par la plante. L'azote est un des principaux facteurs de la croissance des plantes (FAO, 1980). Il est absorbé sous forme de nitrate $\mathrm{NO}_{3}$ - et d'ammonium $\mathrm{NH}_{4}{ }^{+}$par la plante

\section{CONCLUSION}

Les résultats de cette étude indiquent que les valeurs obtenues avec l'Azolla caroliniana étaient statiquement diffèrent de celles des autres fertilisants. La moyenne de (ht) est de 49,37 $\pm 0,55 \mathrm{~cm}$ avec l'Azolla caroliniana contre $37 \pm 1 \mathrm{~cm}$ pour le NPK, $34,57 \pm 2,2$ pour le compost et $22,95 \pm 0,24$ pour le témoin, sauf la croissance $\mathrm{du}(\mathrm{dc})$ où la plus petite différence n'a pas été significative. Le (dc) est de 0,71 $\pm 0,08$; contre 0,57 \pm 0,03 pour le NPK, $0,55 \pm 0,08$ pour le compost et $0,42 \pm$ 0,07 pour le témoin. Le rendement a été important chez l'Azolla caroliniana $(26,13 \pm 0,86$ t/ha de tomate) pour une densité de 37500 plants/ha. A l'issu de
(Layzell, 1990). En plus, azolla libère des minéraux progressivement, ce qui peut assurer leur disponibilité au moment du besoin effectif par la plante. Les éléments nutritifs rendus suffisamment disponibles au fil du temps dans le sol sont efficacement utilisés par les plantes cultivées (Ojetayo et al., 2011). Quant au traitement à base de NPK, nous pouvons attribuer ces résultats à la libération rapide de ces éléments fertilisants (l'azote, le phosphore, et le potassium). L'engrais minéral est utilisé pour corriger rapidement les carences. Cependant, la minéralisation rapide de ce traitement peut entrainer l'infiltration des minéraux dans les horizons inférieurs du sol qui deviennent alors inaccessibles aux racines. Il est donc nécessaire d'y apporter des matières organiques. Cela est justifier par l'idée selon laquelle la matière organique, est le meilleur fertilisant de base (Giller et al., 2002). L'apport d'engrais minéraux seuls ne peut pas maintenir à long terme la productivité des sols à cause du lessivage et de la dégradation des propriétés des sols (Alvarez, 2005). La croissance végétative des tomates traitées avec le compost était moins importante à celle d'azolla et du NPK, car cela s'expliquée selon Bacyé (1993) par le fait que lors de la transplantation à la floraison la matière organique n'était pas décomposée diffusément pour la libération de l'azote nitrique. En plus, l'azote dans le compost est apporté sous forme organique ; ce qui nécessite une transformation de l'azote organique en azote minérale pour une bonne assimilation par les plantes (Cobo et al., 2002). La faible croissance du témoin peut être due aux conditions physico-chimiques du sol. Cette remarque est faite par Mukalay et al. (2008) lorsqu'il affirme que la faible croissance des plantes peut être attribuée aux facteurs caractéristiques du sol, notamment le $\mathrm{pH}$, la toxicité et les déficiences en nutriments (Ca, Mg, P, K, B et Zn).

l'expérimentation, suivant les paramètres considérés, seuls les traitements à base d'azolla excelle en croissance, en hauteur des tiges, au diamètre du collet, en longueur des feuilles et en rendement. Azolla apporté au sol donne une certaine quantité d'éléments nutritifs à la plante et améliore les propriétés physiques du sol. Azolla a démontré ainsi sa capacité de restaurer la fertilité du sol par sa richesse en nutriment capable d'augmenter le rendement de la tomate (Lycopersicum esculentum). L'Azolla peut donc être recommandé aux producteurs de tomate en Côte d'ivoire comme biofertilisant. 


\section{RÉFÉRENCES}

Abidi L.., Snoussi S.A.\& Bradea M.S.., 2017. - Variation du taux de brix sous l'effet d'un Biofertilisant U.P.B. Sci. Bull., Series B, Vol. 79, Iss. 1, ISSN $1454-2331$

Alvarez R., 2005.- A review of nitrogen fertilizer and conservation tillage effects on soil organic carbon storage. Soil Use and Management, 21: 38-52.

Ammari Y., Lamhamedi M.S., Akrimi N. \& El Abidine A.Z., 2006.- Qualités physiologiques de jeunes plants de Pin d'Alep élevés en pépinière moderne sur différents substrats à base de compost. Rev. Geo-Eco-Trop, Volume.30, $n^{\circ} 1,11-24$.

ANADER., 2015.- Document de formation des étudiants UJLOG sur itinéraire technique des cultures maraichère. ANADER zone Daloa.

Anderson J. R., 1992.- Difficulties in Africa agricultural systems enhancement. ten hypotheses agricultural systems, $38: 387-409$.

Anonyme 1., 2013.- Manuel de Formation Statistiques sur les Engrais en Afrique. Disponible sur www. africafertilizer.org.

Anonyme 2., ,2014.- Principaux résultats préliminaires Recensement Général de la Population et de l'Habitat (RGPH). Disponible sur http://www. rgph.org, (dernière consultation mai, 2015)

Bacyé B., 1993.- Influence des systèmes de culture sur l'évolution du statut organique et minéral des ferrugineux et hydromorphes de la zone soudano-sahélienne (Province du Yatenga, Burkina Faso). Thèse doctorat, université d'Aix Marseille III, 243p.

Becking J. H., 1979.- Environmental requirements of Azolla for use in tropical rice production, Nitrogen and Rice. International rice Research Institute. Los Banos, Laguna, Philippines, pp 345-374.

Bernal M.P., Paredes C., Sanchez-Monedero M.A. \& Cegarra J., 1998.- Maturity and stability parameters of composts prepared with a wide range of organic wastes, Bioresource Technologie, Volume. 63, Issue 1, 91-99.

Bikela N. A., 2007.- Essai comparatif de la qualité fertilisante d'Azolla Cristata et d'autres fumures (fiente, lisier et urée). Licence en science, Institut supérieur pédagogique, Gombe Kinshasa, 30p.

Brasset T. \& Couturier C., 2005.- Gestion et valorisation des cendres de chaufferies bois. ADEME, 3p.
Brown M. B. \& Forsythe A. B. 1974.- The small sample behavior of some statistics which test the equality of several means. Technometrics, 16 : 129-132.

Cobo J. G., Barrios E., Kaas D. C. L. \& Thomas R. J., 2002.- Nitrogen mineralization and crop uptake from surface-applied leaves of green manure species on a tropical volcanic-ash soil. Biology and fertility of soils, 36: 87- 92.

Comtois. \& Legaré M.,, 2004.- La Fertilisation des Plantes Ligneuses Cultivées en Contenant. Institut Québécois de Développement de l'Horticulture Ornementale, $57 p$.

Dugué P., 1993.- La gestion de la fertilité et l'utilisation des ressources naturelles dans les systèmes agropastoraux soudano sahéliens. Quelques éléments de réflexion à partir des situations du Yatenga (Burkina Faso) et du Sine Saloum (Sénégal). Communication Cirad n 26 / 93, 15 $\mathrm{p}$.

FAO, 1980.- les engrais et leur application. FAO, Rome, Italie, $51 \mathrm{p}$.

Giller K. E., Cadisch G. \& Palm C., 2002.- The NorthSouth divide : Organic wastes or resources of nutrient management. Agronomy 22: 703-709.

Gnonzan, P. A., 2003.- Valuation of home waste: Influence of constituents report of compost with garbage on the production of the cabbage pomme (Brassica oleracea L.). Graduation report for the engineering degree of works, (SAID), APE/CPU, pp. 75.

Hanson P., Chen J. T., Kuo C. G., Morris R. \& Open R.T. 2001.- Suggestions sur les pratiques culturales de la tomate. Learning center, pp 1 - 9.

Kerkeni A., 2008.- Contribution à la valorisation des composts et des jus de composts : Incidence sur la fertilisation et la protection phytosanitaire de quelques espèces légumières. Thèse Agriculture Durable pour l'obtention du Diplôme de Doctorat en Sciences Agronomiques de I'Institut Supérieur Agronomique de Chott Mariem, Tunisie, $158 p$.

Kitabala M. A., Tshala U. J., Kalenda M. A., Tshijika I. M. \& Mufind K. M., 2016.- Effets de différentes doses de compost sur la production et la rentabilité de la tomate (Lycopersicon esculentum Mill) dans la ville de Kolwezi, Province du Lualaba, RD Congo. Journal of Applied Biosciences, 102: 9669 - 9679. 
Kouadio K. F., 2015.- Contribution des biotechnologies à la sécurité alimentaire: cas du biofertilisant organique (symbiose Anabaena-Azollae, Azolla filiculoides) sur Oryza sativa (riz CB-one) en côte d'Ivoire. Master en science, UFR Agroforesterie, Université Jean Lorougnon Guédé, Daloa, 50p.

Larbi M., 2006.- Influence de la qualité des composts et de leurs extraits sur la protection des plantes contre les maladies fongiques. Thèse de Doctorat de l'Université de Neuchâtel, Suisse, 15-21.

Layzell D. B., 1990.- $\mathrm{N}_{2}$ fixation $\mathrm{NO}_{3}{ }^{-}$reduction and $\mathrm{NH}_{4}{ }^{+}$ assimilation, In : Plant physiology, biochemistry and molecular biology. D.T. Denis and D.H. Teurpin (Eds), Longman scientific \& Technical, Singapore, pp 389-413.

Morel P., Poncet P. \& Rivière L.M., 2000.- Les supports de culture horticoles : Les matériaux complémentaires et alternatifs à la tourbe. Éditions Institut national de la recherche agronomique (INRA), Paris, France, 84 p.

Mukalay M. J., Shutcha M. N., Tshomba K. J., Mulowayi K., Kamb C. F. \& Ngongo L.M., 2008.- Causes d'une forte hétérogénéité des plants dans un champ de maïs dans les conditions pédoclimatique de Lubumbashi. Presses universitaires de Lubumbashi, Annales Faculté des Sciences Agronomiques, vol 1, n² : pp 411

M'sadak Y. \& Ben M'barek A., 2013.- Évaluation de la maturité et de la qualité chimique des substrats de croissance à base de Methacompost avicole pour une meilleure exploitation. Larhyss Journal, $n^{\circ} 23, p p .117-138$.

M'sadak Y. \& Ben M'barek A., 2015.- Caractérisation qualitative du digestat solide de la bio méthanisation industrielle des fientes avicoles et alternative de son exploitation agronomique hors sol. Revue des Énergies Renouvelables, Vol 16 $n^{\circ} 1.33-42$.

Ojetayo A.E., Olaniyi J.O., Akanbi W.B. \& Olabiyi T.I., 2011.- Effect of fertilizer types on nutritional quality of two cabbage varieties before and after storage. Journal of Applied Biosciences 48: 3322-3330.

Sangaré A., Koffi E., Akamou F. \& Fall C. A., 2009.- État des ressources phylogénétiques pour l'alimentation et l'agriculture. Ministère de l'agriculture, Abidjan, République de Côte d'Ivoire, Second rapport. $65 p$
Shapiro S. S., Wilk M. B. \& Chen H. J., 1968.- A comparative study of various tests for normality. J. Am. Statist. Ass. 63: 1343-72.

Singbo G. A., Nouhoheflin T. \& Irissou L. 2004.- Étude des perceptions sur les ravageurs des légumes dans les zones urbaines et périurbaines du sud Bénin. Projet Légumes de qualité, Rapport IITAINRABOBEPAB, Bénin, $21 \mathrm{p}$

Soumaré M., Demeyer A., Tack F.M.G. \& Verloo M.G., 2002.- Chemical Characteristics of Malian and Belgian Solid Waste Composts. Bioresource Technology, Volume. 81, $n^{\circ}$ 2, pp. $97-101$.

Témé B., Breman H. \& Sissoko K. 1995. - Intensification agricole au sahel : mythe ou réalité ? rapport du colloque international sur l'intensification agricole au sahel, Bamako, 28 p. 